\title{
Functional Recovery of the Shoulder after Arthroscopic Treatment for Chronic Calcific Tendinitis
}

\author{
Tae Kyoung Lee, Sang-Jin Shin \\ Department of Orthopedic Surgery, Ewha Womans University Mokdong Hospital, Ewha Womans University College of Medicine, Seoul, Korea
}

\begin{abstract}
Background: We investigated the resolution of pain and functional recovery of shoulder after arthroscopic removal of calcific deposits in patients with chronic calcific tendinitis.

Methods: We enrolled 39 patients who were treated arthroscopically for chronic calcific tendinitis that had been non-responsive to at least 6 months of conservative treatment. We evaluated clinical outcome in terms of the American Shoulder Elbow Surgeons (ASES), the Constant score, the visual analogue score (VAS) for pain. We used plain radiography to measure the size of the calcific deposits. We also analyzed the clinical outcomes in terms of whether or not a cuff repair was performed or the degree of removal of calcific deposits.

Results: We found that complete resolution of pain took on average 5.7 months after the arthroscopic treatment. The ASES and the Constant score significantly improved from the 3-month follow-up, however it took 6 months until the scores reached on average 80 points or above. We found that these clinical outcomes at the final follow-up did not significantly differ by whether or not cuff repair was performed. Similarly, we found that the clinical outcomes did not significantly differ by the degree of calcium removal.

Conclusions: We found that arthroscopic removal of calcification leads to improved clinical outcomes in patients with chronic calcific tendinitis. However, our findings show it takes at least 6 months for the clinical improvement to become statistically significant. We also found that concomitant cuff repairs or the degree of removal of calcification does not affect the clinical outcome of the arthroscopic treatment.
\end{abstract}

(Clin Shoulder Elbow 2018;21(2):75-81)

Key Words: Shoulder joint; Calcific tendinitis; Arthroscopy

\section{Introduction}

Calcific tendinitis of the shoulders is a self-limiting condition, which must pass through formative and resting phases before reaching the resorptive phase. The clinical symptoms of this disease can be classified as either acute or chronic. The resorptive phase of acute calcific tendinitis is known to be the most painful phase, resulting in persistent shoulder pain and restricted range of motion (ROM) ${ }^{1,2)}$ Past studies have shown that acute calcific tendintis is more effectively treated through conservative treatment, such as rest, rehabilitation, and drug therapy, as opposed to surgical treatment, such as arthroscopic removal of calcification. ${ }^{3,4)}$

Chronic calcific tendinitis, however, cannot be treated conservatively with the same favorable outcomes as those of acute calcific tendinitis and often requires surgical removal of calcification for recovery of shoulder function and resolution of pain. Among the surgical treatment methods for chronic calcific tendinitis, the arthroscopic approach has gained popularity because of its association with less shoulder injury and swifter recovery than the standard open approach. In most patients with calcific tendinitis, however, the surgical removal of calcific deposits are performed

Received September 28, 2017. Revised February 14, 2018. Accepted March 11, 2018.

Correspondence to: Sang-Jin Shin

Department of Orthopedic Surgery, Ewha Womans University Mokdong Hospital, Ewha Womans University College of Medicine, 1071

Anyangcheon-ro, Yangcheon-gu, Seoul 07985, Korea

Tel: +82-2-2650-5143, Fax: +82-2-2642-0349, E-mail: sjshin622@ewha.ac.kr, ORCID: https://orcid.org/0000-0003-0215-2860

IRB approval: Ewha Womans University (No. EUMC 2017-10-024-001).

Financial support: This work was supported by the National Research Foundation of Korea (NRF) grant funded by the Korea government (NRF2016R1D1A1A09919541). Conflict of interests: None. 
without sufficient consideration of its consequences. Ark et al. ${ }^{5)}$ have reported that $41 \%$ of patients who receive arthroscopic treatment for chronic calcific tendinitis show intermittent pain for more than 2 years of the treatment. Similarly, in patients with chronic calcific tendinitis, Depalma and Kruper $^{6)}$ noted that arthroscopic treatment is associated with persistent pain as the tendon recovers. There is a debate concerning the surgical treatment of chronic calcific tendinitis on whether cuff lesions induced by the surgical removal of calcific deposits should be repaired or not and whether concomitant subacromial decompression should be performed or not. ${ }^{7-10)}$

In this study, we investigated the clinical outcomes of arthroscopic removal of calcification in patients who were nonresponsive to at least 6 months of conservative treatment for chronic calcific tendinitis. We assessed the improvement in pain and the recovery of shoulder ROM associated with treatment in terms of time-to-recovery. We also investigated whether the clinical outcomes of treatment (postoperative pain and shoulder ROM) were influenced by the intraoperative cuff repair performed after the arthroscopic removal of calcifcation or by the size of the remnant calcification. Here, we found that arthroscopic removal of calcification leads to a significantly satisfactory clinical outcome in patients with chronic calcific tendinitis, but in our study population this effect was observed only after 6 months of follow-up.

\section{Methods}

In our retrospective study, we enrolled 51 patients who were diagnosed with chronic calcific tendinitis and underwent arthroscopic removal of calcific deposits between March 2008 and May 2016. We classified patients as having chronic calcific tendinitis if they were symptomatic for more than 3 months and were non-responsive or had worsened symptoms after six months of conservative treatment. Types of conservative treatment patients received included non-steroidal anti-inflammatory drug (NSAID) medication, physiotherapy, subacromial injection, and extracorporeal shockwave therapy (ESWT). The following inclusion criteria were used: 1) recalcitrant pain despite at least 6 months of conservative treatment; 2) follow-up period of 1 year; 3) a preoperative calcific deposit of greater than $5 \mathrm{~mm}$ (determined through plain radiography); and 4) calcific tendinitis confined to the supraspinatus tendon (determined through magnetic resonance imaging $[\mathrm{MRI}])$. The exclusion criteria were one or more of the following: symptomatic calcific tendinits of fewer than 3 months, acute calcific tendinitis, fewer than 6 months of conservative treatment, calcific tendinitis of the subscapularis tendon or the infraspinatus tendon, and follow-up of less than 1 year. We also excluded patients with preoperative MRI findings that revealed presence of concomitant shoulder lesions (such as cuff tears), osteoarthritis, infections, or tumors and patients with a history of shoulder surgery of the ipsilateral arm or cervical or neurological abnormalities. Informed consent was received from all patients. The study was approved by the Institutional Review Board of Ewha Womans University (EUMC 2017-10-024-001).

We conducted arthroscopic removal of the calcific deposits in the lateral position. After making a general assessment of the intraarticular lesion, we moved the arthroscope through the posterior portal into the subacromial space. Then through the anterior portal, we performed capsular release to expose the rotator cuff and the calcific deposits. In case the calcific deposits could not be clearly located on the cuff surface, we made a puncture with a spinal needle at the site predicted on the preoperative MRI scan to be the site of calcification. We used a motorized shaver to remove the calcific deposits, removing as much deposits as possible whilst keeping the cuff intact. However, if this process led to a cuff deficit greater than $2 \mathrm{~cm}$ or thicker than $70 \%$ of the cuff width, we conducted a single-screw cuff repair. When radiographic and arthroscopic findings showed signs of cuff erosion or subacromial impingement, we performed acromioplasty. After the surgery, all patients were administered an arm sling for approximately 3 weeks. They were instructed to perform the passive ROM exercises on their own. If passive ROM induced pain in patients, oral NSAIDs were administered. Neither ESWT nor sono-guided needling was performed.

We conducted follow-up examinations of our patients at the following three time points: 3 months, 6 months, and 12 months postoperatively. To evaluate size, location, and resorption of the calcific deposits, we conducted plain radiography in the anteroposterior, axillary, and supraspinatus outlet views before and after the surgical treatment (at each follow-up).We conducted preoperative MRI to assess the presence of concomitant diseases. At each follow-up, we assessed the passive ROM of the affected shoulder with a full-circle manual goniometer, measuring anterior flexion, abduction, external rotation (at $0^{\circ}$ of abduction), and internal rotation behind the back. The handbehind-back method was used to measure the highest point of spinal segment reached by the tip of the extending thumb with the hand facing out, and for statistical analysis the hand-behindback range was measured on a continuous scale of 0 and 90 . The spinal scale began from the sacral segment (0) and ended at the 9th thoracic segment (90). Each articulation of the spinal segment in between also corresponded to specific point on the scale (e.g., the 5th lumber segment, 10; the 1st lumber segment, 50; the 12th thoracic segment, 60).

We assessed the clinical and radiological outcomes of treatment. Shoulder pain was assessed preoperatively and postoperatively with visual analogue score (VAS) for pain. We defined complete resolution of pain as a VAS score of lower than one. Shoulder function was assessed at the same time-points in terms 
of the American Shoulder Elbow Surgeons (ASES) score and the Constant score. We analyzed treatment outcome by whether or not cuff repair was performed and whether the calcific deposit was completely or incompletely removed.

\section{Statistical Analysis}

We used the IBM SPSS ver. 21.0 program (IBM Co., Armonk, NY, USA) for all statistical analyses. To compare the difference between the pre- and postoperative clinical parameters, we performed the Wilcoxon signed rank test. To assess whether the clinical outcome differed in terms of whether a cuff repair was performed concomittantly or whether the calcific deposits were completely or incompletely removed, we used the MannWhitney test. A $p<0.05$ was considered statistically significant.

\section{Results}

We excluded 12 of 51 patients from our data analysis for the following reasons: lost to follow-up (9 patients) and calcific tendinitis locating to the subscapularis tendon (3 patients). Our final study population consisted of 39 patients (12 male and 27 female patients). The average age of the patients was $54.1 \pm 7.7$ years. The demographic characteristics of the patients are summarized in Table 1.

In all patients, we found that VAS for pain was significantly improved after athroscopic decompression (from 7.9 at the initial follow-up to 0.3 at the final follow-up, $p<0.001)$. However, pain completely resolved in patietns only on average $5.7 \pm 3.1$ months postoperatively (after 3 months in 2 patients and after 6 months in 31 patients). Two patients whose VAS for pain remained above 3 points even until the 12-month follow-up were treated conservatively, through subacromial steroid injection and drug therapy. We also found that the ASES score and the Constant score significantly improved from the 3 -month followup through to the final follow-up in all patients ( $p<0.001$; Fig. 1 ). Yet the improvement was slow, taking 6 months before the average score reached 80 points.

We found that although the four passive ROMs assessed

Table 1. Patient Demographics

\begin{tabular}{lc}
\hline Gender (male:female) & $12: 27$ \\
\hline Arm dominance (\%) & 74.3 \\
\hline Patient age (yr) & $54.1 \pm 7.7$ \\
\hline Symptom duration (mo) & $31.3 \pm 34.4$ \\
Mean follow-up period (mo) & $12.3 \pm 8.8$ \\
\hline Preoperative intraarticular steroid injection (times) & $2.5 \pm 4.5$ \\
Size of calcific deposit (mm) & $13.5 \pm 5.7$ \\
\hline
\end{tabular}

Values are presented as mean \pm standard deviation unless otherwise noted. in this study were significantly improved by the final followup, none showed a statistically significant improvement at the 3-month follow-up (Table 2). Statistically significant improvements were observed later on; abduction, external rotation, and internal rotation, at the 6-month follow-up and anterior flexion, at the 12-month follow-up.

We conducted an intraoperative cuff repair for 13 patients whose rotator cuff was damaged because of the arthroscopic treatment. We found that whether or not patients received a cuff repair clinical parameters significantly improved with arthroscopic treatment at the final follow-up. Neither demographic data nor clinical outcome significantly differed between the two groups (Table 3).

Using plain radiography, we found that the calcific deposits significantly decreased in size with arthroscopic treatment (from $13.5 \pm 5.7 \mathrm{~mm}$ preoperatively to $3.5 \pm 4.0 \mathrm{~mm}$ postoperatively; $p<0.001$ ). We found that the clinical outcomes did not depend on the degree of calcium removal because the clinical outcomes between the 17 patients whose calcific deposits were completely removed and the rest whose calcific deposits were incompletely removed did not differ at the final follow-up (Table 4). In the latter group, radiographic measurement of calcific resorption revealed that the calcific deposits were completely resorbed. In these patients, the average time to complete resorption of calcification from treatment was $5.2 \pm 1.9$ months.

\section{Discussion}

In this study, we found that arthroscopic removal of calcific deposits in patients with chronic tendinitis resolves pain and restores shoulder function. We found that the degree of improvement in our patients was moderate until the 3-month followup and that these improvements only became prominent from

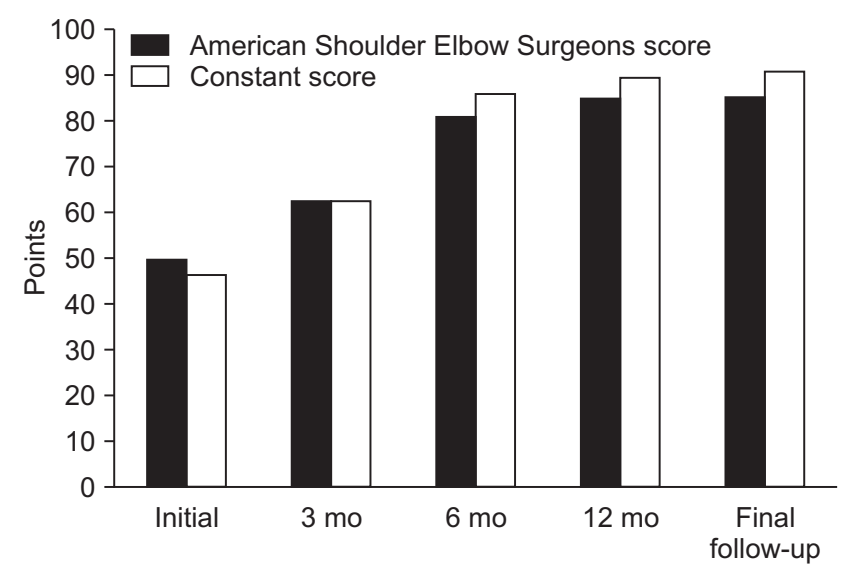

Fig. 1. Clinical outcomes at each follow-up. All clinical outcomes significantly improved at the final follow-up in comparison to their respective preoperative values. 
Table 2. Serial Change in Passive Range of Motion over the Postoperative Recovery Period

\begin{tabular}{|c|c|c|c|c|}
\hline \multirow{2}{*}{ Variable } & \multicolumn{4}{|c|}{ Passive range of motion $\left(^{\circ}\right)$} \\
\hline & Forward flexion & Abduction & External rotation & Internal rotation \\
\hline Initial & $162.5 \pm 22.7$ & $154.3 \pm 25.3$ & $62.5 \pm 13.9$ & $59.2 \pm 12.0$ \\
\hline POD 3 months & $163.0 \pm 23.0$ & $155.1 \pm 26.8$ & $63.5 \pm 15.4$ & $60.3 \pm 12.8$ \\
\hline$p$-value & 0.16 & 0.71 & 0.21 & 0.21 \\
\hline POD 6 months & $166.1 \pm 18.2$ & $158.4 \pm 25.7$ & $64.1 \pm 14.9$ & $61.8 \pm 12.3$ \\
\hline$p$-value & 0.08 & $0.01^{*}$ & $0.01^{*}$ & $0.01^{*}$ \\
\hline POD 12 months & $170.0 \pm 11.0$ & $162.8 \pm 17.9$ & $66.4 \pm 10.6$ & $64.9 \pm 9.1$ \\
\hline$p$-value & $0.02^{*}$ & $0.001^{\star}$ & $0.01^{*}$ & $0.001^{*}$ \\
\hline Last visit & $172.3 \pm 9.8$ & $166.1 \pm 16.0$ & $67.4 \pm 10.4$ & $65.1 \pm 9.1$ \\
\hline$p$-value & $0.001^{*}$ & $<0.001^{*}$ & $<0.001^{*}$ & $<0.001^{*}$ \\
\hline
\end{tabular}

Values are presented as mean \pm standard deviation. All $p$-values were compared to the initial range of motion.

POD: postoperative day.

${ }^{\star}$ Statistically significant compared to initial range of motion.

Table 3. Clinical Outcomes According to Concomitant Rotator Cuff Repair after Arthroscopic Calcification Removal

\begin{tabular}{|lccc}
\hline \multicolumn{1}{|c}{ Variable } & $\begin{array}{c}\text { Concomitant } \\
\text { rotator cuff repair } \\
(\mathrm{n}=13)\end{array}$ & $\begin{array}{c}\text { Decompression } \\
\text { only } \\
(\mathrm{n}=26)\end{array}$ & -value \\
\hline Mean age (yr) & $54.9 \pm 6.4$ & $53.8 \pm 8.5$ & 0.74 \\
\hline Gender (male:female) & $2: 11$ & $10: 16$ & \\
\hline Symptom duration (mo) & $35.5 \pm 44.5$ & $29.1 \pm 61.4$ & 0.73 \\
\hline Size of calcific materials (mm) & $14.3 \pm 7.2$ & $13.0 \pm 5.2$ & 0.67 \\
\hline VAS score & & & \\
\hline Preoperative & $7.9 \pm 0.8$ & $7.8 \pm 0.7$ & 0.80 \\
\hline Final follow-up & $0.5 \pm 0.9$ & $0.5 \pm 0.7$ & 0.78 \\
\hline ASES score & & & \\
\hline Preoperative & $50.8 \pm 11.0$ & $49.2 \pm 14.6$ & 0.80 \\
\hline Final follow-up & $84.5 \pm 9.3$ & $85.0 \pm 6.7$ & 0.85 \\
\hline Constant score & & & \\
\hline Preoperative & $53.2 \pm 13.3$ & $48.7 \pm 15.8$ & 0.38 \\
\hline Final follow-up & $90.5 \pm 4.3$ & $89.2 \pm 6.0$ & 0.76 \\
\hline
\end{tabular}

Values are presented as mean \pm standard deviation or number only.

VAS: visual analogue score, ASES: American Shoulder Elbow Surgeons.

the 6-month follow-up and onwards. We also found that factors such as concomitant cuff repair or size of remnant calcification did not influence the clinical outcome.

Generally, symptomatic calcific tendinitis can be conservatively treated with satisfactory clinical outcomes and improvement of symptoms. Those that do not achieve improvement of symptoms despite 6 months of conservative treatment are called chronic calcific tendinitis. The spontaneous resorption of calcium in chronic calcific tendinitis do not occur, and unlike acute
Table 4. Clinical Outcomes According to the Degree of Calcification Removal

\begin{tabular}{lccc}
\hline \multicolumn{1}{c}{ Variable } & $\begin{array}{c}\text { Incomplete } \\
\text { removal }(\mathrm{n}=22)\end{array}$ & $\begin{array}{c}\text { Complete } \\
\text { removal }(\mathrm{n}=17)\end{array}$ & $p$-value \\
\hline Size of calcific desposit $(\mathrm{mm})$ & & & \\
\hline Preoperative & $15.6 \pm 6.3$ & $10.9 \pm 3.8$ & $0.01^{*}$ \\
\hline Immediate postoperative & $6.1 \pm 3.7$ & $0.2 \pm 0.3$ & $<0.001^{*}$ \\
\hline VAS score & & & \\
\hline Preoperative & $7.8 \pm 0.7$ & $7.8 \pm 0.7$ & 0.94 \\
\hline Final follow-up & $0.6 \pm 0.9$ & $0.2 \pm 0.4$ & 0.21 \\
\hline ASES score & & & \\
\hline Preoperative & $47.7 \pm 14.8$ & $52.3 \pm 11.3$ & 0.60 \\
\hline Final follow-up & $83.7 \pm 8.2$ & $86.3 \pm 6.6$ & 0.38 \\
\hline Constant score & & & \\
\hline Preoperative & $48.9 \pm 15.8$ & $51.9 \pm 14.1$ & 0.36 \\
\hline Final follow-up & $89.0 \pm 5.5$ & $90.5 \pm 5.5$ & 0.20 \\
\hline
\end{tabular}

Values are presented as mean \pm standard deviation.

VAS: visual analogue score, ASES: American Shoulder Elbow Surgeons.

${ }^{\star}$ Statistically significant.

calcific tendinitis this condition is often resistant to conservative treatment. ${ }^{11)}$ Because conservative treatment, especially those for the treatment of resistant conditions, tend to be longer and more associated with recurrences than surgical treatment, it can lead to prolonged pain and restrictions in shoulder function. ${ }^{12)}$ Sono-guided needling, a common treatment method for chronic calcific tendinitis, has been shown to be associated with positive long-term outcomes but not with complications. ${ }^{13,14)}$ However, it has been shown that among patients who received sono-guided needling $25 \%$ to $45 \%$ require revision treatment and $17 \%$, arthroscopic treatment. ${ }^{15,16)}$ Seil et al. ${ }^{17)}$ have recommended that 
chronic calcific tendinitis that are resistant to conservative treatment should be treated as early as possible with more aggressive treatment.

Calcific deposits in chronic calcific tendinitis are removed to restore shoulder function and alleviate pain in the patients. Here, we found that $94 \%$ of patients with chronic calcific tendinitis showed complete resolution of pain at the 12-month follow-up; however, the time taken to achieve complete resolution of pain was relatively long, taking at least 6 months for a significant improvement to show in the majority of our patients. Similarly, Depalma and Kruper $^{6}$ ) $r e p o r t e d$ that at least a 10-week recovery period is needed after treatment for calcifying tendinitis. Studies have shown that postoperative pain may occur as tendon is reconstructed at the site of tissue injury after complete or incomplete removal of calcification. ${ }^{18)}$ Therefore, it is imperative to inform patients before treatment that pain may persist up to 6 months of the treatment.

We found that the difference in the ROMs measured between the preoperative examination and the 3-month followup was not statistically significant; this suggests that functional recovery did not occur at the early stages of recovery. Maier et al. $^{2,19)}$ suggested that causes of restricted shoulder function and delayed functional recovery after treatment in patients with chronic calcific tendinitis may be prolonged pain or postoperative complications, such as cuff tears or subacromial impingement, emphasizing therefore the importance of rehabilitation. In this study, approximately $82 \%$ of patients showed a significant improvement in shoulder function only after 6 months of treatment, amongst whom two still had restricted shoulder ROM at the one-year follow-up. These findings imply that postoperative rehabilitation should be performed over a sufficiently long period, lasting at least 6 months after surgical treatment.

Conflicting opinions on the degree of removal of calcification during the arthroscopic treatment of chronic calcific tendinitis exist because the more calcification is removed the greater the possibility of cuff damage. For instance, Jerosch et al. ${ }^{20)}$ argued for the complete removal of calcification as their findings showed that arthroscopic removal of calcific deposits leads to statistically significant favorable outcomes. Unlike Jerosch et al., ${ }^{20)}$ our results show that the degree of removal and the clinical outcome at the final follow-up was not correlated, suggesting that complete arthroscopic removal is not necessary. Some studies have suggested that it is sufficient to simply confirm the complete resorption of calcification at the recovery stage (i.e., during the follow-up period) in patients who received an incomplete removal of calcification for symptom relief and for the prevention of recurrences. ${ }^{21)}$ In this study, although 17 of 39 patients had incomplete removal of calcification (the relatively high proportion of incomplete removals is because the study's surgical approach prioritized keeping the cuff intact and therefore preferentially used the compression method), only two patients had non-resolved pain at the 6-month follow-up. Our findings suggest that complete arthroscopic removal of calcific deposits is not necessary for chronic calcific tendinitis.

In this study, we found that the incomplete removal group had on average a 6-mm calcific deposit left immediately after the surgery. Although the value was larger than the 5-mm threshold size used in this study for indication of surgical treatment, the patients nevertheless showed a significantly decreased amount of calcification compared to pre-treatment levels. Multiple drilling performed during partial removal of calcification would have lowered the internal pressure of the rotator cuff; we anticipate that this would have promoted resorption of calcification during the recovery period. In fact, the final follow-up findings revealed that the remnant calcification had been completely resorbed in all the patients. The postoperative spontaneous resorption of the remnant calcification may explain the similarity in clinical outcome between patients whose calcifc deposits were completely removed and those whose were not. In this study, most remnant calcification in patients after the arthroscopic treatment presented as a paste-like appearance deep within the rotator cuff and completely attached, meaning that if we had attempted to remove the calcification completely by surgical means, the surgical treatment may have induced a complete cuff tear and postoperative complications, such as pain. Thus, we suggest that calcific deposits should be removed as much as possible but without damaging the rotator cuff. However, remnant calcification that is $15 \mathrm{~mm}$ or larger has been shown to be associated with a more frequent recurrence of acute pain. ${ }^{22)}$ None of the patients in this study had remnant calcification exceeding this size and, therefore, did not report of any recurrences during the follow-up period. Nevertheless, if there is still much remnant calcification left after an arthroscopic treatment for chronic calcific tendinitis, follow-up radiography should be regularly performed to evaluate the remnant calcification.

There is no consensus concerning the repair of cuff ruptures caused by arthroscopic removal of calcific deposits in the current literature, and some studies have reported that there are no clear indications for a cuff repair after arthroscopic removal of calcification. However, Porcellini et al. ${ }^{23)}$ have reported that patients show better improvement in pain when cuff lesions larger than $1 \mathrm{~cm}$ are repaired than when they are not repaired. In line with their study, we conducted cuff repairs if the region of cuff deficit was larger than $2 \mathrm{~cm}$ or encompassed more than $70 \%$ of the cuff width (that is, if it was a full-thickness tear). Because cuff repairs may lead to secondary stiffness, we performed early rehabilitation and shoulder exercises in 13 of 39 patients in this study to prevent secondary complications and to promote ROM recovery. All patients except one showed good clinical outcome.

Several limitations to this study exist. We used a retrospec- 
tive study design, meaning that our analyses of data are based primarily on patient records, and a relatively small study population. The study design did not include a control treatment, such as a conservative treatment (e.g., sono-guided needling), that would have enabled us to demonstrate the superiority of the surgical method over the conservative method. Moreover, we did not investigate whether the type of cuff repair (simple sideto-side repair vs. anchor repair) influences clinical outcome. Because our study used a relatively short-term follow-up, the findings of our study cannot exclude the possibility of re-tears or recurrence of symptoms in the long-term; therefore, it is necessary to conduct studies with longer follow-ups to exclude longterm complications.

\section{Conclusion}

Here, we found that the clinical outcomes of arthroscopic removal of calcific deposits were not affected by whether not or cuff repair was performed or by the degree of calcific removal, complete or incomplete, in patients with recalcitrant chronic calcific tendinitis. Our findings show that although arthroscopic treatment of calcific tendinitis improves shoulder function and alleviates pain in these patients at least a 6-month period is needed before these clinical improvements can be observed.

\section{References}

1. Uhthoff HK. Calcifying tendinitis. Ann Chir Gynaecol. 1996; 85(2):111-5.

2. Maier D, Jaeger M, Izadpanah K, Suedkamp NP, Ogon P. Arthroscopic treatment of calcific tendinitis of the shoulder. Am J Sports Med. 2012;40(7):NP12-3; author reply NP13.

3. Balke M, Bielefeld R, Schmidt C, Dedy N, Liem D. Calcifying tendinitis of the shoulder: midterm results after arthroscopic treatment. Am J Sports Med. 2012;40(3):657-61.

4. Rowe CR. Calcific tendinitis. Instr Course Lect. 1985;34:1968.

5. Ark JW, Flock TJ, Flatow EL, Bigliani LU. Arthroscopic treatment of calcific tendinitis of the shoulder. Arthroscopy. 1992;8(2): 183-8.

6. Depalma AF, Kruper JS. Long-term study of shoulder joints afflicted with and treated for calcific tendinitis. Clin Orthop. 1961;20:61-72.

7. Clement ND, Watts AC, Phillips C, McBirnie JM. Short-term outcome after arthroscopic bursectomy debridement of rotator cuff calcific tendonopathy with and without subacromial decompression: a prospective randomized controlled trial. Arthroscopy. 2015;31(9):1680-7.

8. Maier D, Jaeger M, Izadpanah K, Bornebusch L, Suedkamp NP, Ogon P. Rotator cuff preservation in arthroscopic treatment of calcific tendinitis. Arthroscopy. 2013;29(5):824-31.
9. Ranalletta M, Rossi LA, Bongiovanni SL, Tanoira I, Piuzzi N, Maignon G. Arthroscopic removal and rotator cuff repair without acromioplasty for the treatment of symptomatic calcifying tendinitis of the supraspinatus tendon. Orthop J Sports Med. 2015;3(4):2325967115577957.

10. Yoo JC, Park WH, Koh KH, Kim SM. Arthroscopic treatment of chronic calcific tendinitis with complete removal and rotator cuff tendon repair. Knee Surg Sports Traumatol Arthrosc. 2010;18(12):1694-9.

11. Brewer BJ. Aging of the rotator cuff. Am J Sports Med. 1979; 7(2):102-10.

12. Ozkoç G, Akpinar S, Hersekli MA, Ozalay M, Tandoğan RN. Arthroscopic treatment of rotator cuff calcifying tendinitis. Acta Orthop Traumatol Turc. 2002;36(5):413-6.

13. Kim YS, Lee HJ, Kim YV, Kong CG. Which method is more effective in treatment of calcific tendinitis in the shoulder? Prospective randomized comparison between ultrasound-guided needling and extracorporeal shock wave therapy. J Shoulder Elbow Surg. 2014;23(11):1640-6.

14. Serafini G, Sconfienza LM, Lacelli F, Silvestri E, Aliprandi A, Sardanelli F. Rotator cuff calcific tendonitis: short-term and 10-year outcomes after two-needle us-guided percutaneous treatment: nonrandomized controlled trial. Radiology. 2009;252(1):157-64.

15. Louwerens JK, Veltman ES, van Noort A, van den Bekerom MP. The effectiveness of high-energy extracorporeal shockwave therapy versus ultrasound-guided needling versus arthroscopic surgery in the management of chronic calcific rotator cuff tendinopathy: a systematic review. Arthroscopy. 2016;32(1):16575.

16. Yoo JC, Koh KH, Park WH, Park JC, Kim SM, Yoon YC. The outcome of ultrasound-guided needle decompression and steroid injection in calcific tendinitis. J Shoulder Elbow Surg. 2010;19(4):596-600.

17. Seil R, Litzenburger H, Kohn D, Rupp S. Arthroscopic treatment of chronically painful calcifying tendinitis of the supraspinatus tendon. Arthroscopy. 2006;22(5):521-7.

18. Uhthoff HK, Loehr JW. Calcific tendinopathy of the rotator cuff: pathogenesis, diagnosis, and management. J Am Acad Orthop Surg. 1997;5(4):183-91.

19. Maier D, Jaeger M, Izadpanah K, et al. Arthroscopic removal of chronic symptomatic calcifications of the supraspinatus tendon without acromioplasty: analysis of postoperative recovery and outcome factors. Orthop J Sports Med. 2014;2(5): 2325967114533646.

20. Jerosch J, Strauss JM, Schmiel S. Arthroscopic treatment of calcific tendinitis of the shoulder. J Shoulder Elbow Surg. 1998;7(1):30-7.

21. Jacobs R, Debeer P. Calcifying tendinitis of the rotator cuff: functional outcome after arthroscopic treatment. Acta Orthop Belg. 2006;72(3):276-81. 
22. Bosworth DM. Diagnosis and treatment of lesions of the shoulder. R I Med J. 1953;36(9):512-5.

23. Porcellini G, Paladini P, Campi F, Paganelli M. Arthroscopic treatment of calcifying tendinitis of the shoulder: clinical and ultrasonographic follow-up findings at two to five years. J Shoulder Elbow Surg. 2004;13(5):503-8. 